\title{
Recursive partitioning analysis of patients with oligometastatic non-small cell lung cancer: a retrospective study
}

\author{
Jia-Tao Zhang, Si-Yang Liu, Hong-Hong Yan, Yi-Long Wu, Qiang Nie* and Wen-Zhao Zhong ${ }^{*}$ (D)
}

\begin{abstract}
Background: Local consolidative treatment (LCT) is important for oligometastasis, defined as the restricted metastatic capacity of a tumor. This study aimed to determine the effects and prognostic heterogeneity of LCT in oligometastatic non-small cell lung cancer.

Methods: This retrospective study identified 436 eligible patients treated for oligometastatic disease at the Guangdong Provincial People's Hospital during 2009-2016. A Cox regression analysis was used to identify potential predictors of overall survival (OS). After splitting cases randomly into training and testing sets, risk stratification was performed using recursive partitioning analysis with a training dataset. The findings were confirmed using a validation dataset. The effects of LCT in different risk groups were evaluated using the Kaplan-Meier method.

Results: The T stage ( $p=0.001)$, N stage $(p=0.008)$, number of metastatic sites $(p=0.031)$, and EGFR status $(p=0.043)$ were identified as significant predictors of OS. A recursive partitioning analysis was used to establish a prognostic risk model with the following four risk groups: Group I included never smokers with N0 disease (3-year OS: 55.6\%, median survival time [MST]: 42.8 months), Group II included never smokers with N+ disease (3-year OS: 32.8\%, MST: 26.5 months), Group III included smokers with T0-2 disease (3-year OS: 23.3\%, MST: 19.4 months), and Group IV included smokers with T3/4 disease (3-year OS: 12.5\%, MST: 11.1 months). Significant differences in OS according to LCT status were observed in all risk groups except Group IV ( $p=0.45)$.
\end{abstract}

Conclusions: Smokers with T3/4 oligometastatic non-small cell lung cancer may not benefit from LCT.

Keywords: Oligometastasis, Local consolidative therapy, Recursive partitioning analysis, Prognosis risk stratification

\section{Background}

A comprehensive review of metastasis theory before 1995 reveals two main mechanisms, Halsted's theory and the systemic hypothesis, which were addressed using local and systemic treatment, respectively [1-4]. In 1995, Hellman and Weichselbaum proposed a clinically significant state of metastasis, "oligometastasis," which refers to a restricted tumor metastatic capacity [5]. This clinical entity was initially considered an intermediate state of metastatic evolution, and local treatments were considered potentially curative in this setting [6-8]. However, the precise definition of oligometastasis remains

\footnotetext{
* Correspondence: bulaier6480@163.com; 13609777314@163.com Guangdong Lung Cancer Institute, Guangdong Provincial People's Hospital, Guangdong Key Laboratory of Lung Cancer Translational Medicine, South China University of Technology \& Guangdong Academy of Medical Sciences, Guangzhou 510080, China
}

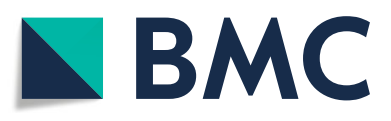

(c) The Author(s). 2019 Open Access This article is distributed under the terms of the Creative Commons Attribution 4.0 International License (http://creativecommons.org/licenses/by/4.0/), which permits unrestricted use, distribution, and reproduction in any medium, provided you give appropriate credit to the original author(s) and the source, provide a link to the Creative Commons license, and indicate if changes were made. The Creative Commons Public Domain Dedication waiver (http://creativecommons.org/publicdomain/zero/1.0/) applies to the data made available in this article, unless otherwise stated.

highly uncertain, which ultimately affects our understanding of the mechanisms underlying cancer metastasis. However, a diagnosis of oligometastasis mainly relies on the observation of a change after systemic therapy, and the number of metastatic sites (either $1-3$ or $1-5$ ) is considered the main determinant.

Lung cancer is the leading cause of cancer-related death worldwide, and more than 700,000 new cases are diagnosed each year in China $[9,10]$. Over the past decade, studies concerning oligometastasis have identified various factors that can predict a favorable prognosis and support an indication for local therapy [7, 11-13]. The first randomized phase 2 study on this topic, which investigated local consolidative therapy (LCT) for oligometastatic non-small cell lung cancer (NSCLC), was reported in 2016 and revealed median progression-free 
survival (PFS) durations of 11.9 months in the LCT group and 3.9 months in the maintenance treatment group [14]. In other words, LCT appears useful for improving the prognosis of patients with oligometastasis, although several problems (e.g., predictive factors and treatment timing) must be resolved before this approach can be implemented in clinical practice.

Previous studies have indicated that the stable progression-free period after first-line systemic therapy may be the optimal window for LCT. Therefore, the present study aimed to evaluate the prognostic heterogeneity and factors related to LCT in patients with oligometastatic NSCLC.

\section{Methods}

This retrospective study evaluated data from patients with oligometastatic NSCLC who were treated at Guangdong Provincial People's Hospital. The retrospective study protocol was approved by the ethics committee of Guangdong Provincial People's Hospital.

\section{Patient selection and LCT definition}

In this report, we define oligometastasis as stage IV disease with $\leq 3$ metastases, not including the primary tumor, based on the 7th edition of the TNM system. And the detailed inclusion criteria as follow: (1) pathologically confirmed NSCLC, (2) stage IV disease based on the 7th TNM staging system, (3) $\leq 3$ synchronous or metachronous metastases (not including the primary tumor), (4) an Eastern Cooperative Oncology Group performance status of $\leq 2$, and (5) a history of first-line systemic therapy ( $\geq 2$ cycles of platinum-based chemotherapy or $\geq 1$ month of EGFR/ALK targeted therapy). The number of metastatic sites was assessed using systemic imaging, namely computed tomography (CT), electrical capacitance tomography, or positron emission tomography (PET)-CT of the chest and abdomen and $\mathrm{CT}$, magnetic resonance imaging, or PET-CT of the brain. Patients with pleural, pericardial, and meningeal metastases were excluded because the metastatic lesions could not be counted separately.

The decision to perform LCT was made by a panel of clinicians, including a thoracic surgeon, radiologist, and medical oncologist. LCT was defined as treatment with the intent to ablate all residual disease (primary tumor, lymph nodes, and metastatic sites) comprising surgery, radiotherapy, or both. The treating radiotherapists made decisions about any dose-fractionation regimen with curative intent when possible, although palliative intent was considered acceptable.

\section{Study design}

A random number was assigned to each case according to the principle of simple randomization. Based on the numerical order of the random numbers, patients in the first half were grouped as the training set; the remaining patients were grouped as the validation set. A recursive partitioning analysis (RPA) based on the patients' demographic and clinical characteristics was then performed with the intent to create a decision tree model that would correctly stratify risk in the target population [15]. The model was subsequently evaluated using the validation dataset. The effects of LCT were also investigated in various risk groups.

Table 1 Baseline characteristics from the training and validation datasets

\begin{tabular}{|c|c|c|c|}
\hline & Training & Validation & $P$-value \\
\hline Age, years (range) & $60.4(36-88)$ & $60.4(31-84)$ & 0.331 \\
\hline Sex, n (\%) & & & 0.045 \\
\hline Male & $164(75.2 \%)$ & $144(66.1 \%)$ & \\
\hline Female & $54(24.8 \%)$ & 74 (33.9\%) & \\
\hline Smoking history, n (\%) & & & 0.101 \\
\hline Never & $112(51.4 \%)$ & $130(59.6 \%)$ & \\
\hline Former/current & $106(48.6 \%)$ & $88(40.4 \%)$ & \\
\hline Metastatic sites, n (\%) & & & 0.233 \\
\hline $1-2$ & $208(95.4 \%)$ & $201(92.2 \%)$ & \\
\hline 3 & $10(4.6 \%)$ & $17(7.8 \%$ & \\
\hline Classification, n (\%) & & & 0.690 \\
\hline Synchronous & $137(62.8 \%)$ & $142(65.1 \%)$ & \\
\hline Metachronous & $81(37.2 \%)$ & $76(34.9 \%)$ & \\
\hline Pathology, n (\%) & & & 0.616 \\
\hline Adenocarcinoma & $164(75.2 \%)$ & $171(78.4 \%)$ & \\
\hline Non-adenocarcinoma & $47(21.6 \%)$ & $45(20.6 \%)$ & \\
\hline Unknown & $7(3.2 \%)$ & $2(1.0 \%)$ & \\
\hline EGFR status, n (\%) & & & 0.911 \\
\hline Negative & $110(50.5 \%)$ & 107 (49.1\%) & \\
\hline Positive & 77 (35.3\%) & $82(37.6 \%)$ & \\
\hline Unknown & $31(14.2 \%)$ & $29(13.3 \%)$ & \\
\hline T stage, n (\%) & & & 0.851 \\
\hline TO-2 & $149(68.3 \%)$ & $155(71.1 \%)$ & \\
\hline T3-4 & 67 (30.7\%) & $61(27.9 \%)$ & \\
\hline N stage, n (\%) & & & 0.282 \\
\hline No & $49(22.5 \%)$ & $63(28.9 \%)$ & \\
\hline $\mathrm{N}+$ & $169(77.5 \%)$ & $155(71.1 \%)$ & \\
\hline LCT & & & 0.968 \\
\hline Surgery & $17(7.8 \%)$ & $15(6.9 \%)$ & \\
\hline Radiotherapy & $79(36.2 \%)$ & $80(36.7 \%)$ & \\
\hline Both & $4(1.8 \%)$ & $3(1.4 \%)$ & \\
\hline Neither & $118(54.2 \%)$ & $120(55.0 \%)$ & \\
\hline Total & 218 & 218 & \\
\hline
\end{tabular}

EGFR epidermal growth factor receptor; $L C T$ local consolidative therapy 


\section{Statistical methods}

Associations between clinical characteristics were evaluated using the chi-square test. The Kaplan-Meier method and log-rank test were used to evaluate differences in overall survival (OS) and PFS. For the RPA, the recursive decision tree was created using free software ( $\mathrm{R}$ version 3.3.2; rpart package version 4.1-11, http:// www.r-project.org/) and was pruned by complexity parameter.

\section{Results}

This study included 436 patients with oligometastatic NSCLC who were treated during 2009-2016. The baseline characteristics from the training and validation datasets are shown in Table 1. The two datasets only differed significantly in terms of sex. Figure 1 presents the results from the Cox regression analysis of all patients, which revealed associations of an inferior outcome with the number of metastatic sites (hazard ratio [HR]: 1.91, $p=0.031$ ), T stage (HR: $2.36, p<0.001$ ), and $\mathrm{N}$ stage (HR: 2.25, $p<0.001$ ). Furthermore, an improved outcome was associated with EGFR mutation (HR: 0.67, $p=0.043)$.

The results of the RPA model for OS are shown in Fig. 2a. Based on the training dataset, the patients were divided into four risk groups: Group I included never smokers with N0 disease (3-year OS: 83.1\%), Group II included never smokers with $\mathrm{N}+$ disease (3-year OS: 28.7\%), Group III included smokers with T0-2 disease (3-year OS: 28.0\%), and Group IV included smokers with T3/4 disease (3-year OS: 18.3\%). The various risk groups had significantly different 3 -year OS rates $(p<0.001)$. The survival curves for the RPA model in the training and validation datasets are shown in Fig. $2 \mathrm{~b}$ and c, respectively.

Figure 3 presents the clinical characteristics of all patients according to the RPA model and the survival curves of each risk group according to LCT status. Significant differences in the survival curves according to LCT status were observed for all risk groups except Group IV $(p=0.45)$, indicating that LCT provided limited survival benefits in this group of patients with oligometastatic NSCLC.

\section{Discussion}

A considerable amount of literature has been dedicated to oligometastasis, and the importance of LCT in this context has gradually been accepted [16-21]. However, various problems must be addressed before specific patients with oligometastatic NSCLC who are expected to benefit from LCT can be identified. Therefore, the present study used RPA to examine the effects and prognostic heterogeneity of LCT for oligometastatic NSCLC. Our results indicate that patients who smoked and had T3/4 oligometastatic NSCLC would not be expected to benefit from LCT. However, the EGFR status was the only molecular characteristic considered in this study. As 60 patients (13.8\%) did not undergo the related test, we cannot definitively comment on the contributions of molecular features in this group of patients. Moreover, in clinical practice, patients with a history of smoking and a large lung tumor typically have squamous cell

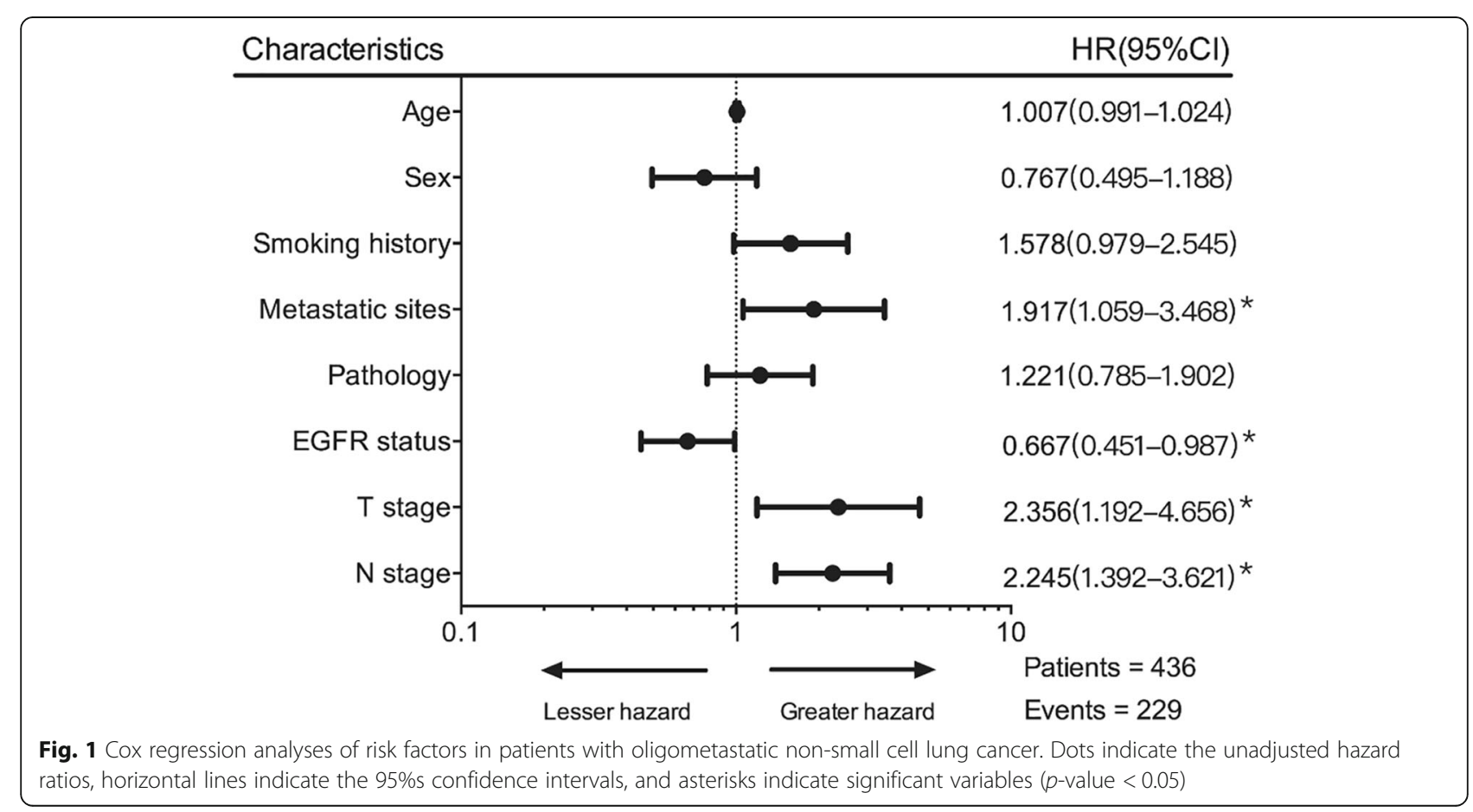




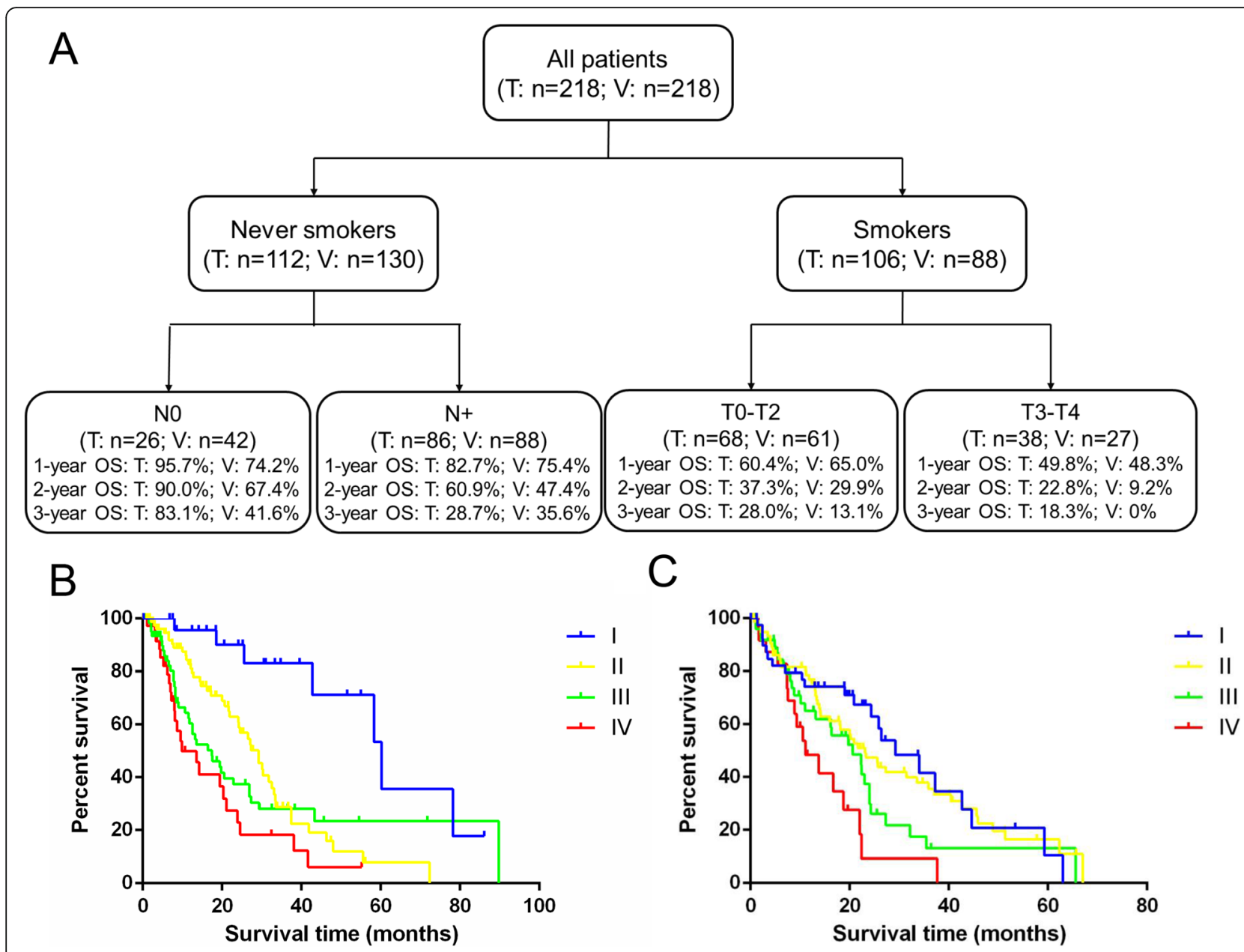

Fig. 2 Flowchart of the recursive partitioning analysis. a The recursive partitioning analysis of patients with oligometastatic non-small cell lung cancer ( $T$ : training set, $V$ : validation set). $\mathbf{b}$ The survival curves for the training dataset. $\mathbf{c}$ The survival curves for the validation dataset

carcinoma or a tumor without driver gene mutations, which suggests the presence of an unknown molecular mechanism that should be examined in future studies.

As shown in Table 1, sex characteristics were slightly imbalanced between the two datasets $(p=0.045)$. However, after careful consideration, we chose to accept this slight imbalance for the following reasons: 1) the relevant factors that could be affected by gender, such as pathology, EGFR status, and smoking history, were completely balanced between the two groups; 2) gender was not a statistically significant factor in the Cox regression model; 3) we believed that the slight gender difference would be acceptable and the subsequent RPA model could be verified in the two datasets; and 4) we used simple randomization in this study, which is likely to lead to this type of issue.

At the 60th ASTRO annual meeting, Professor Gomez presented the final results of a phase 2 trial that compared LCT to standard maintenance treatment or observation alone for patients with oligometastatic NSCLC.
During a median follow-up period of 38.8 months (range: 28.3-61.4 months), the median OS was significantly longer in the LCT group than in the control group (41.2 months [95\% CI: 18.9 months-not reached] vs. 17.0 months [10.1-39.8 months]; $p=0.017$ ) [22]. Interestingly, in our study, patients in Group IV had a median OS of 11.1 months, similar to that in the control group. Conversely, Group I had a median OS (42.8 months) similar to that in the LCT group. These preliminary results support our main conclusion that patients in our Group IV (smokers with T3/4 disease) may not benefit from LCT.

The present study excluded patients with pleural, pericardial, and meningeal metastasis because these metastases could not be counted separately. Moreover, evidence suggests that pleural or meningeal metastases are disease entities with unique biological behaviors. For example, Zhong et al. reported that patients with intrathoracic disseminated pT4-M1a pleural metastases had a favorable prognosis [23]. In addition, several studies have indicated that limited surgery might be a good choice for 


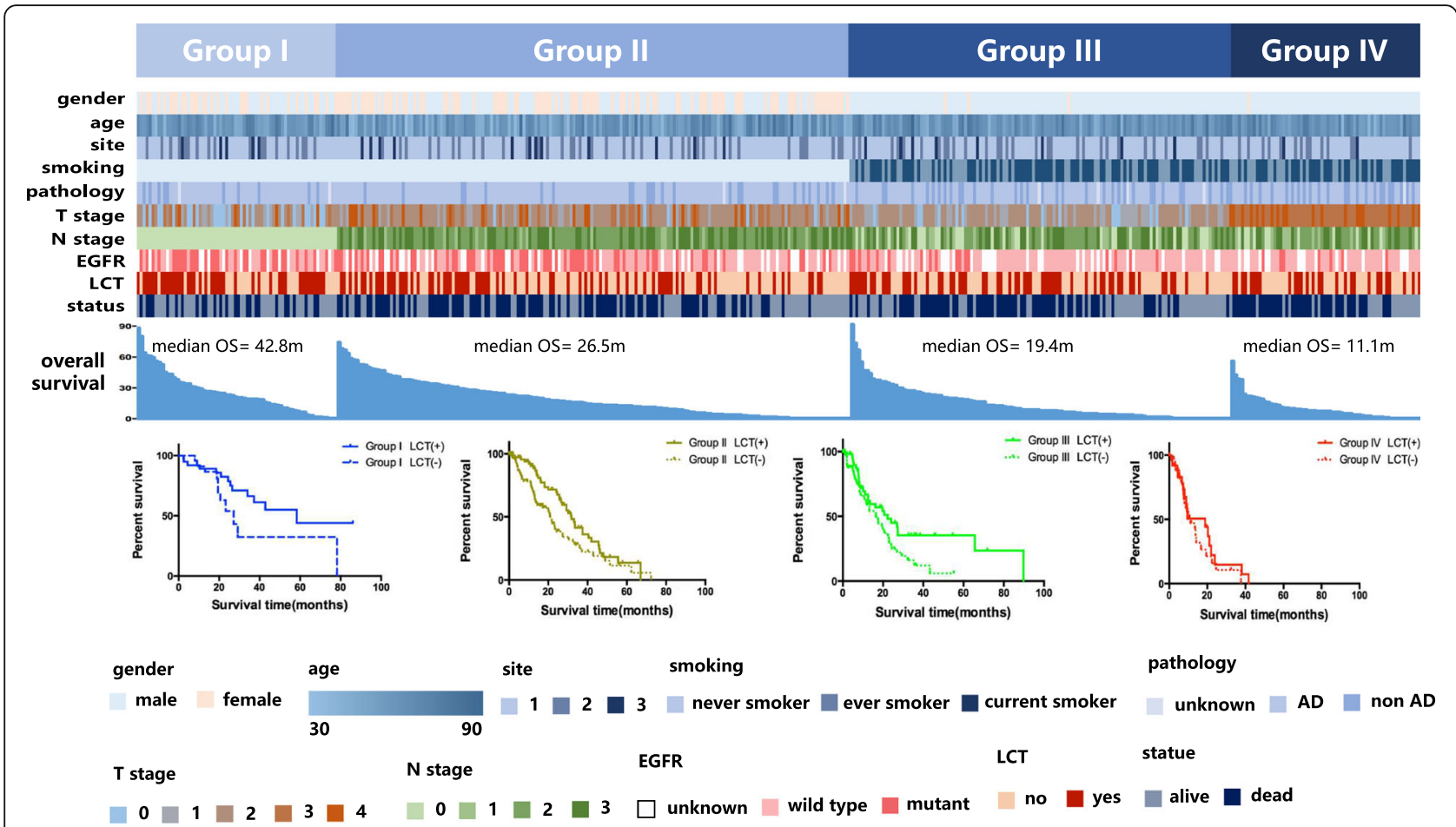

Fig. 3 Distribution of patients' characteristics according to the recursive partitioning analysis and the effects of local consolidative treatment in different risk groups

the pleural dissemination of lung cancer [20, 24, 25]. However, meningeal metastasis, and especially leptomeningeal metastasis, is a different disease entity with a poor prognosis. Few studies have investigated the comprehensive profile of meningeal metastasis, for which no treatment strategies have been established. Li et al. recently reported that leptomeningeal metastasis was much more common in patients with EGFR-mutant NSCLC, who responded relatively well to EGFR-TKIs [26]. Therefore, we believe that our exclusion of patients with pleural, pericardial, and meningeal metastasis was reasonable.

The present study had two major limitations. First, a retrospective design is associated with a risk of selection bias. The selection of LCT for oligometastatic patients may have been an additional source of bias. Second, censoring the data may have confounded the results of our analyses. Moreover, we intended to base the RPA tree on the most obvious prognostic differences, which could have introduced some degree of ambiguity in the categorical variables. Nevertheless, we believe that our findings may enable clinical oncologists to better select LCT for patients with oligometastatic NSCLC. We have launched a phase II study to further explore the role of LCT for oligometastatic NSCLC after first-line systemic treatment; this study commenced in 2018 (Chinese Thoracic Oncology Group, CTONG 1602).

\section{Conclusions}

In conclusion, we used the results of our single-center study to create a stratification model that would predict the effects of LCT on oligometastatic NSCLC. The results indicate that smokers with $\mathrm{T} 3 / 4$ oligometastatic NSCLC may not benefit from LCT. However, future studies are needed to explore the genetic signatures of patients who may benefit from LCT for oligometastatic disease.

\section{Abbreviations}

LCT: Local consolidative treatment; NSCLC: Non-small cell lung cancer; OS: Overall survival; PFS: Progression-free survival

\section{Acknowledgements \\ None.}

Authors' contributions

Conceived and designed the study: JTZ, SYL. Collected and analyzed the data: JTZ, SYL, HHY. Wrote the paper: JTZ. Reviewed the paper: QN, WZZ, YLW. All authors have read and approved the manuscript.

\section{Funding}

This research was supported by funding from the National Natural Science Foundation of China (81673031, 81872510), the Guangzhou Municipal Science and Technology Grant (201704020161). These funding bodies did not play any roles in the study design, data collection and analysis, or decision to publish the findings.

Availability of data and materials

The datasets used and/or analyzed during the current study are available from the corresponding author on reasonable request. 


\section{Ethics approval and consent to participate}

The protocol of this retrospective study was approved by the ethics committee of Guangdong Provincial People's Hospital.

\section{Consent for publication}

Not applicable.

\section{Competing interests}

The authors declare that they have no competing interests.

Received: 14 February 2019 Accepted: 30 September 2019

Published online: 06 November 2019

\section{References}

1. Reyes DK, Pienta KJ. The biology and treatment of oligometastatic cancer. Oncotarget. 2015;6:8491-524.

2. Weigelt B, Peterse JL, van't Veer LJ. Breast cancer metastasis: markers and models. Nat Rev Cancer. 2005;5:591-602.

3. Halsted WS. I. the results of radical operations for the cure of carcinoma of the breast. Ann Surg. 1907:46:1-19.

4. Tait CR, Waterworth A, Loncaster J, Horgan K, Dodwell D. The oligometastatic state in breast cancer: hypothesis or reality. Breast. 2005; 14:87-93.

5. Hellman S, Weichselbaum RR. Oligometastases. J Clin Oncol. 1995:13:3.

6. Weichselbaum RR, Hellman S. Oligometastases revisited. Nat Rev Clin Oncol. 2011:8:378-82

7. Barton MK. Local consolidative therapy may be beneficial in patients with oligometastatic non-small cell lung cancer. CA Cancer J Clin. 2017:67:89-90,

8. Congedo MT, Cesario A, Lococo F, De Waure C, Apolone G, Meacci E, et al. Surgery for oligometastatic non-small cell lung cancer: long-term results from a single center experience. J Thorac Cardiovasc Surg. 2012;144:444-52.

9. Chen W, Zheng R, Baade PD, Zhang S, Zeng H, Bray F, et al. Cancer statistics in China, 2015. CA Cancer J Clin. 2016;66:115-32.

10. Siegel RL, Miller KD, Jemal A. Cancer statistics, 2018. CA Cancer J Clin. 2018:68:7-30

11. Ashworth AB, Senan S, Palma DA, Riquet M, Ahn YC, Ricardi U, et al. An individual patient data metaanalysis of outcomes and prognostic factors after treatment of oligometastatic non-small-cell lung cancer. Clin Lung Cancer. 2014;15:346-55.

12. David EA, Clark JM, Cooke DT, Melnikow J, Kelly K, Canter RJ. The role of thoracic surgery in the therapeutic management of metastatic non-small cell lung cancer. J Thorac Oncol. 2017;12:1636-45.

13. Endo C, Hasumi T, Matsumura Y, Sato N, Deguchi H, Oizumi H, et al. A prospective study of surgical procedures for patients with oligometastatic non-small cell lung cancer. Ann Thorac Surg. 2014;98:258-64.

14. Gomez DR, Blumenschein GR Jr, Lee JJ, Hernandez M, Ye R, Camidge DR, et al. Local consolidative therapy versus maintenance therapy or observation for patients with oligometastatic non-small-cell lung cancer without progression after first-line systemic therapy: a multicentre, randomised, controlled, phase 2 study. Lancet Oncol. 2016;17:1672-82.

15. Ciampi A, Lawless JF, McKinney SM, Singhal K. Regression and recursive partition strategies in the analysis of medical survival data. J Clin Epidemiol. 1988:41:737-48

16. Tree AC, Khoo VS, Eeles RA, Ahmed M, Dearnaley DP, Hawkins MA, et al. Stereotactic body radiotherapy for oligometastases. Lancet Oncol. 2013;14:e28-37.

17. Sheu T, Heymach JV, Swisher SG, Rao G, Weinberg JS, Mehran R, et al, Propensity score-matched analysis of comprehensive local therapy for oligometastatic non-small cell lung cancer that did not progress after frontline chemotherapy. Int J Radiat Oncol Biol Phys. 2014;90:850-7.

18. Sastry P, Tocock A, Coonar AS. Adrenalectomy for isolated metastasis from operable non-small-cell lung cancer. Interact Cardiovasc Thorac Surg. 2014;18:495-7.

19. Pfannschmidt J, Dienemann $\mathrm{H}$. Surgical treatment of oligometastatic nonsmall cell lung cancer. Lung Cancer. 2010;69:251-8.

20. Okamoto T, Iwata T, Mizobuchi T, Hoshino H, Moriya Y, Yoshida S, et al. Pulmonary resection for lung cancer with malignant pleural disease first detected at thoracotomy. Eur J Cardiothorac Surg. 2012;41:25-30.

21. Lo SS, Moffatt-Bruce SD, Dawson LA, Schwarz RE, Teh BS, Mayr NA, et al. The role of local therapy in the management of lung and liver oligometastases. Nat Rev Clin Oncol. 2011;8:405-16.
22. Gomez DR, Tang C, Zhang J, Blumenschein GR, Hernandez M. Local consolidative therapy (LCT) improves overall survival (OS) compared to maintenance therapy/observation in oligometastatic non-small cell lung cancer (NSCLC): final results of a multicenter, randomized, controlled phase 2 trial: 60th ASTRO Annual Meeting; 2018.

23. Zhong WZ, Li W, Yang XN, Liao RQ, Nie Q, Dong S, et al. Accidental invisible intrathoracic disseminated pT4-M1a: a distinct lung cancer with favorable prognosis. J Thorac Dis. 2015;7:1205-12.

24. Shimizu J, Oda M, Morita K, Hayashi Y, Arano Y, Matsumoto I, et al. Comparison of pleuropneumonectomy and limited surgery for lung cancer with pleural dissemination. J Surg Oncol. 1996:61:1-6.

25. Fukuse T, Hirata T, Tanaka F, Wada H. The prognostic significance of malignant pleural effusion at the time of thoracotomy in patients with nonsmall cell lung cancer. Lung Cancer. 2001;34:75-81.

26. Li YS, Jiang BY, Yang JJ, Tu HY, Zhou Q, Guo WB, et al. Leptomeningeal metastases in patients with NSCLC with EGFR mutations. J Thorac Oncol. 2016;11:1962-9.

\section{Publisher's Note}

Springer Nature remains neutral with regard to jurisdictional claims in published maps and institutional affiliations.

Ready to submit your research? Choose BMC and benefit from:

- fast, convenient online submission

- thorough peer review by experienced researchers in your field

- rapid publication on acceptance

- support for research data, including large and complex data types

- gold Open Access which fosters wider collaboration and increased citations

- maximum visibility for your research: over $100 \mathrm{M}$ website views per year

At BMC, research is always in progress.

Learn more biomedcentral.com/submissions 